\title{
Inflammatory response of thymus bystander effects on acute radiation-induced skin injury in rats
}

\author{
Y.T. Chen ${ }^{1,2,3^{*}}$, C.C. Li ${ }^{1,2,3^{*}}$, Y. Liu ${ }^{1,2,3}$, Y.Q. Zheng4, X.H. Yang5, \\ C.X. Yang1,2,3, M.W. An ${ }^{1,2,3^{*}}$ \\ ${ }^{1}$ Institute of Biomedical Engineering, College of Biomedical Engineering, Taiyuan University of Technology, \\ Taiyuan, Shanxi, China \\ ${ }^{2}$ Shanxi Key Laboratory of Material Strength \& Structural Impact, Taiyuan University of Technology, Taiyuan, \\ Shanxi, China \\ ${ }^{3}$ Research Center for Nanobiomaterials \& Regenerative Medicine, College of Biomedical Engineering, Taiyuan \\ University of Technology, Taiyuan, Shanxi, China \\ ${ }^{4}$ Centre of Radiation Oncology, Shanxi Cancer Hospital, Taiyuan, Shanxi, China \\ ${ }_{5}^{5}$ Shanxi Provincial Cancer Institute Laboratory Animal Center, Shanxi Cancer Hospital, Taiyuan, Shanxi, China
}

\section{- Original article}

\section{*Corresponding authors:}

Meiwen An, PhD.,

E-mail: meiwen_an@163.com

Revised: June 2020

Accepted: June 2020

Int. J. Radiat. Res., April 2021; 19(2): 409-419

DOI: $10.29252 /$ ijrr.19.2.409

\begin{abstract}
Background: Radiation not only kills tumor cells, but also damages other sites. The mechanisms of damage caused by the bystander effect of irradiation in animal models are unclear and the time node is single. In this study, we aimed to investigate the inflammatory response of thymus tissue injury in non-irradiated areas at different times after irradiating rat skin. Materials and Methods: Rats were irradiated with an X-ray dose of $38 \mathrm{~Gy}$, and at $15 \mathrm{~d}$ after irradiation, when the skin wound was most severe, the proinflammatory drug high mobility group box1 (HMGB1) and the antiinflammatory drug glycyrrhizic acid (GA) were injected intraperitoneally into rats. After irradiation, skin tissues were collected for histology, and thymus tissues were collected for gene and protein testing. Results: Animal model of skin damage was successfully established. The expression of macrophage (F4/80) increased after irradiation, and F4/80 produced cytokines. Through the flow which was activated by inflammatory factors in the blood, DNA damage and the expression of inflammatory-related cytokines in nonirradiated area of the thymus peaked at $15 \mathrm{~d}$ after irradiation. Moreover, HMGB1 treatment increased the expression at $1 \mathrm{~d}$ after intraperitoneal injection, and GA solution decreased the expression of inflammatory-related cytokines. Conclusion: When radiation damages the skin, it can cause damage to other organs through the circulation, and an anti-inflammatory GA solution reduced inflammatory responses, which are required to modify radiationinduced systemic effects with anti-inflammatory drugs or agents that affect pathways that cause bystander instability.
\end{abstract}

Keywords: Radiation induced bystander effects, thymus, $\mathrm{YH}_{2} A X, N L R P_{3}, H M G B 1$.

\section{INTRODUCTION}

With the development and progression of science and technology, ionizing radiation has been widely used in the diagnosis and treatment of various diseases. In the past, it was believed that DNA damage that was directly or indirectly induced by ionizing radiation was the only cause of biological processes, including apoptosis and carcinogenesis (1). In 1992, Nagasawa et al. (2) irradiated Chinese hamster ovary cells with $\alpha$-particles, and found that $30 \%$ of the cells showed chromatid exchange, however less than $1 \%$ of the cells was irradiated by $\alpha$-particles. 
These findings indicated that similar ionizing radiation damage occurred in cells around irradiated cells that were not directly irradiated. In cells that were not directly irradiated, processes including cell death, gene mutations, and chromosome instability occurred, which were similar to the processes found in cells that were directly exposed to radiation. We named these effects radiation-induced bystander effects (RIBEs) (3).

The thymus is an important part of the immune system and is very sensitive to radiation. There is evidence that DNA damage caused by oxidative stress is a key factor in the development of RIBEs. Furthermore, after a high dose of radiation, the body's immune cells will contain DNA double-strand breaks (DSBs), triggering point mutations, and other processes, resulting in increased cell membrane permeability, and even cell death $(4,5)$. DSBs are the most lethal (6), and the most serious RIBEs (7), and gamma histone family $2 \mathrm{~A}$ variant $\left(\gamma \mathrm{H}_{2} \mathrm{AX}\right)$ foci have been found as molecular markers of DSBs (8). Anderson et al. (9) found that ionizing radiation can cause serious damage to the immune system and lymphocytes. Moreover, Xin et al (10) confirmed that radiation can cause low immune function of the thymus and a dysfunctional secretion of immune cytokines.

Radiation damage to the body can also produce a series of inflammatory responses through the circulation. In 2002, Martinon et al. (11) first identified a crucial protein in inflammatory responses, named the "inflammasome", among which the NOD-like receptor family, pyrin domain-containing protein $3\left(\mathrm{NLRP}_{3}\right)$ was one of the most widely and deeply studied one (12). The inflammasome was made up of $\mathrm{NLRP}_{3}$, apoptosis-associated speck-like protein containing a CARD (ASC), and caspase-1. Moreover, $\mathrm{NLRP}_{3}$ is also an important factor in early inflammatory responses. High mobility group box1 (HMGB1) is an important inflammatory factor in the late inflammatory response. After binding with receptor for advanced glycation end products (RAGE) receptors, nuclear factor kappa B (NF-KB) signaling was activated by activation of cell divisioncycle 42/ractopamine hydrochloride (CDC42/Rac), mitogen-activated protein kinase (MAPK), p38MAPK or extracellular regulated protein kinases1/2 (ERK1/2) to release inflammatory factors and promote acute inflammatory responses (13). Recombinant HMGB1 solution is a proinflammatory drug that has been used to promote inflammatory responses. Glycyrrhizic acid (GA) is an anti-inflammatory drug, which can inhibit the occurrence of inflammation and has been applied in clinical practice. Khan et al. (14) demonstrated that oxygen-free radicals produced by radiation-induced inflammatory cytokines, such as tumor necrosis factor (TNF) and interleukin-1 (IL-1), can lead to indirect DNA damage in non-irradiated areas. Moreover, in vitro studies have shown that the bystander mechanism can lead to changes in the hematopoietic microenvironment, and that macrophage-mediated cytotoxic inflammatory responses in the exposed tissues may also be a reason of delayed DNA damage (15). Jung et al. (16) found that the inflammasome was a key regulator of HMGB1-induced inflammation, and they believed that HMGB1 was involved in inflammatory responses by promoting the activation of $\mathrm{NLRP}_{3}$, the inflammasome, and interleukin-1 beta (IL-1 $\beta$ ). On the other hand, once DNA damage responses were prolonged and repair fails, dead cells will release DNA-HMGB1 to induce a second wave of inflammatory responses (17).

To the best of our knowledge, most of the published studies on the damage caused by the bystander effect of irradiation were based on the cell level or tissue level, and in only a few studies, the results animal models were used. Therefore, the primary aim of the present study was to explore the inflammatory response and influence of radiation on distant organs in rats using a relatively stable animal model of acute radioactive skin injury, thereby providing a reference for the safety of clinical applications. The secondary aim was to investigate whether anti-inflammatory drugs can reduce the injury of distant organs, thereby providing a theoretical basis for the use of clinical drugs. 


\section{MATERIALS AND METHODS}

Forty-two male Wistar rats, eight to nine-week-old weighing 280-320 g were purchased from the Experimental Animal Center of the Shanxi cancer hospital [SCXK (Jing) 2016-0001]. All experimental rats were kept on a $12 \mathrm{~h}$ light/dark schedule in a single cage with free access to water and food. The experimental program had been approved by the animal ethics committee of the Shanxi cancer hospital (ethical review approval number: GDY2018001) (Shanxi, China). All animal experiments were conducted according to the operational guidelines of the Experimental Animal Center of the Shanxi cancer hospital (Shanxi, China) and the Regulations on the Management of Experimental Animals of the People's Republic of China.

\section{Drugs and irradiation}

Rats were randomly divided into seven groups of six rats per group. Group 1 was a control group, and the surgery was identical as that of rats in groups 2-7, except rats did not undergo irradiation. Rats in group 2-7 were exposed to X-irradiation with a dose of $38 \mathrm{~Gy}$ at a dose rate of $300 \mathrm{cGy} \cdot \mathrm{min}^{-1}$ via the $6 \mathrm{MV}$ photon beam of a medical electron linear accelerator (Siemens, Germany). The irradiation field was a circular region with a diameter of $3 \mathrm{~cm}$ that was given at the root of the right posterior buttock. The source skin distance (SSD) was $100 \mathrm{~cm}$, and the unexposed area was shielded by lead. At $15 \mathrm{~d}$ after irradiation, rats in group 6 were intraperitoneally injected with $20 \mu \mathrm{g}$ of the pro-inflammatory drug HMGB1 $\left(20 \mu \mathrm{g} \cdot \mathrm{mL}^{-1}\right.$, in normal saline) (eBioscience, USA) and rats in group 7 were intraperitoneally injected with $10 \mathrm{mg} \cdot(\mathrm{kg} \cdot \mathrm{d})^{-1}$ of the anti-inflammatory drug glycyrrhizic Acid (GA) (2mg. $\mathrm{mL}^{-1}$, in normal saline) (Solarbio, Beijing, China). Skin tissues were collected and fixed in an embedding box with 4\% paraformaldehyde for paraffin embedding and immunohistochemistry. Thymus issues were placed in a sterile enzyme-free cryopreservation tube for real time quantitative PCR (qRT-PCR) and Western blot analysis.

\section{Hematoxylin and Eosin (HE) Staining}

The collected rat skin samples were fixed, dehydrated, transparent, wax-permeable and embedded, then sliced continuously on a pathological microtome (Leica instruments, Shanghai, China). After baking in a $60^{\circ} \mathrm{C}$ oven, sections were put into tissue paraffin in xylene I (20 min) (Sinopharm, China), xylene II dewaxing (20 min) (Sinopharm, China), then in gradient wine, distilled water was used to clean. Finally, hematoxylin and eosin (HE) staining (Solarbio, Beijing, China) was performed to dehydrate the sections and seal the coverslip. Skin sections stained with HE were examined using an optical microscope (IX70, Olympus, Japan).

\section{Immunofluorescence}

Paraffin sections were dewaxed in water, and placed in a repair box filled with EDTA antigen repair buffer (Servicebio, Wuhan, China). Next, sections were placed in a microwave oven (Microwave oven appliance, Galanz, China) for 8 min at medium fire $-8 \mathrm{~min}$ at ceasefire $-7 \mathrm{~min}$ at low fire. Bovine serum albumin (BSA) (Servicebio, Wuhan, China) was added, then sections were incubated overnight with the primary antibody (1:1000, Abcam, China) at $4^{\circ} \mathrm{C}$. Sections were washed and incubated with the secondary antibody (1:1000, Abcam, China). Finally, sections were incubated with 4',6diamidino-2-phenylindole (DAPI) (Servicebio, Wuhan, China). Anti-fluorescent quenching agent (Servicebio, Wuhan, China) was added in to seal the coverslip. Stained sections were placed under an inverted fluorescence microscope (Nikon, Japan) for observation and image collection.

\section{Immunohistochemical analysis}

Paraffin sections were dewaxed in water, and the antigen was retrieved, then it was immersed in hydrogen peroxide solution in the dark for 25 min. BSA (Servicebio, Wuhan, China) was added. Then the primary and secondary antibodies (1:1000, Abcam, China) were added. Diaminobenzidine (DAB) (Servicebio, Wuhan, China) chromogen was added, and after the chromogen was developed, development was 
stopped by washing with tap water. Sections were incubated with hematoxylin (Servicebio, Wuhan, China) for 3 min, washed with tap water, then washed with hematoxylin differentiation solution (Servicebio, Wuhan, China) for $5 \mathrm{~s}$, and then added with hematoxylin blue returning solution (Servicebio, Wuhan, China). Finally, sections were dehydrated and sealed, observed under a light microscope (Nikon, Japan), and photographed for image collection.

\section{Real-time PCR}

The primer sequences used in this study were designed, synthesized, and purchased from Sangon Biological Engineering Company (table 1). An RNA extraction kit (Servicebio, Wuhan, China) was used to extract RNA from tissues according to the manufacturer's instructions. Total RNA of 2 microngrams was taken and reversely transcribed into complementary DNA (cDNA) using a reverse transcription kit (Servicebio, Wuhan, China). According to the instructions of the kit, PCR reaction was conducted with $1 \mu \mathrm{L}$ cDNA strand as a template, with 0.15 primers, in a total volume of 20 microns. The PCR reaction was as follows: 10 min at $95^{\circ} \mathrm{C}$, then denaturation at $95^{\circ} \mathrm{C}$ for $15 \mathrm{~s}$, annealing at $60^{\circ} \mathrm{C}$ for $30 \mathrm{~s}$, extension at $72^{\circ} \mathrm{C}$ for $10 \mathrm{~min}$, with a total of 40 cycles. The amplification curve and dissolution curve were automatically generated by Applied Biosystems Step0ne Plus Software v2.1. The results were analyzed by the comparative threshold cycle (Ct) method and were normalized to the expression of GAPDH.

\section{Western Blot analysis}

Thymus tissues were thoroughly homogenized by homogenizer (KZ-II, Kangtao Technology, China), then centrifuged at 12000 rpm for $2 \mathrm{~min}$, and the supernatant was the total protein. The protein concentration of the tissue samples was determined by the bicinchoninic acid (BCA) (Servicebio, Wuhan, China) method. Separate glue and concentrated glue were prepared, loaded onto the gel and electrophoresis was performed. The film was turned with wet rotation. The primary antibody
(1:1000, Abcam, China) was added and incubated overnight at $4^{\circ} \mathrm{C}$. Washed with TBST for three times and $10 \mathrm{~min}$ for one time. Then secondary antibody (1:1000, Abcam, China) was added and incubated at $37^{\circ} \mathrm{C}$ for $2 \mathrm{~h}$. Then the film was exposed. After taking images, Image Plus 5.1 software was used to measure the average optical density of the bands, phase quantitative analysis was conducted, and the ratio of target proteins to internal parameters was calculated for statistical analysis. For loading control, $\beta$-actin was assessed.

\section{Statistical analysis}

Experimental values were expressed as the mean value \pm standard deviation $(\bar{x} \pm S D)$. The charts were created using Origin Pro 8.5 software, and SPSS 22.0 software was used for statistical analyses. One-way Anova was used for comparison between groups. $\mathrm{P}<0.05$ was considered statistically significant.

Table 1. qRT-PCR primers.

\begin{tabular}{|c|c|c|}
\hline \begin{tabular}{|c|} 
Gene \\
name
\end{tabular} & Primer Sequence ( $5^{\prime}$ to $\left.3^{\prime}\right)$ & \begin{tabular}{|c|} 
PCR product \\
size (bp)
\end{tabular} \\
\hline \multirow{2}{*}{$\gamma \mathrm{H}_{2} \mathrm{AX}$} & F CCTCACTGCCGAGATCCTGGAG & \multirow{2}{*}{105} \\
\hline & R TCCTCGTCGTTGCGGATAGCC & \\
\hline \multirow{2}{*}{$\mathrm{NLRP}_{3}$} & F TCTTTGCGGCTATGTACTATCT & \multirow{2}{*}{113} \\
\hline & R TTCTAATAGGACCTTCACGT & \\
\hline \multirow{2}{*}{ HMGB1 } & F ACAACACTGCTGCGGATGACAAG & \multirow{2}{*}{177} \\
\hline & R CCTCCTCGTCGTCTTCCTCTTCC & \\
\hline \multirow{2}{*}{ GAPDH } & F ACGGCAAGTTCAACGGCACAG & \multirow{2}{*}{129} \\
\hline & R CGACATACTCAGCACCAGCATCAC & \\
\hline
\end{tabular}

\section{RESULTS}

\section{Irradiated skin: appearance and HE-staining}

Macroscopic observation showed that within the second week after irradiation, the skin in the exposed area of the rats was red, swollen, and hyperemic. In the third week after irradiation, the skin was damaged, and the damage was the most significant at about $15 \mathrm{~d}$ after irradiation (figure 1A). HE-staining results showed that the epidermis of non-irradiated skin tissues was intact, and the epithelial cells had a normal appearance, and were closely arranged. There Int. J. Radiat. Res., Vol. 19 No. 2, April 2021 
were hair follicles, sebaceous glands, and other accessory organs in the cortex. No obvious inflammation was observed. Collagen arrangement in the dermis was loose. Both the epidermis and dermis at $6 \mathrm{~h}-48 \mathrm{~h}$ after irradiation were clear in structure, clear in boundary, and in the epidermis. There were hair follicles, sebaceous glands, and other accessory organs in the dermis, however, no obvious inflammation was observed. Large areas of epidermal necrosis were seen at $15 \mathrm{~d}$ after irradiation. A small amount of inflammatory cell infiltration was seen in the dermis (yellow arrow) (figure 1B). Local thickening of the epidermis and hyperplasia of epithelial cells (red arrow) (Figure 1B) can be seen at $18 \mathrm{~d}$ after irradiation. Collagen was increased in the dermis, with a small amount of inflammatory cell infiltration (yellow arrow) (Figure 1B). Sebaceous glands were not seen in the dermis, which indicated that the skin was severely

A

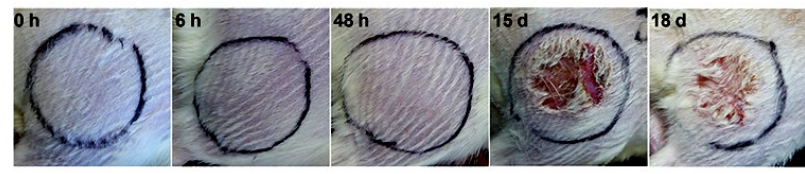

B

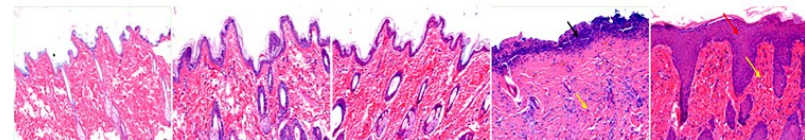

Figure 1. Skin of the right buttock of a rat (A) and hematoxylin and eosin (HE)-staining results (B) (magnification $\times 100$ ) between the $X$-ray group and the control group at different times after irradiation.

damaged after irradiation (figure 1B).

\section{The expression of macrophages (F4/80) in} irradiated skin

In normal skin tissues, F4/80-positive cells were expressed in only a few hair follicles and other skin appendices (figure 2A). At $6 \mathrm{~h}$ after irradiation, many $\mathrm{F} 4 / 80$ positive cells were distributed in the deep dermis (figure 2A). At 48 $\mathrm{h}$ after irradiation, the number of $\mathrm{F} 4 / 80$ positive cells in the deep dermis decreased, however, its expression peaked (figure 2B). At $15 \mathrm{~d}$ after irradiation, F4/80-positive cells were almost all distributed in the dermal tissue (figure 2A). At
$18 \mathrm{~d}$ after irradiation, a small number of F4/80-positive cells was distributed in the epidermal tissue, and the green fluorescence intensity was very low. No F4/80-positive cells were observed in the dermis (figure $2 \mathrm{~A}$ ), thereby indicating that F4/80 expression was

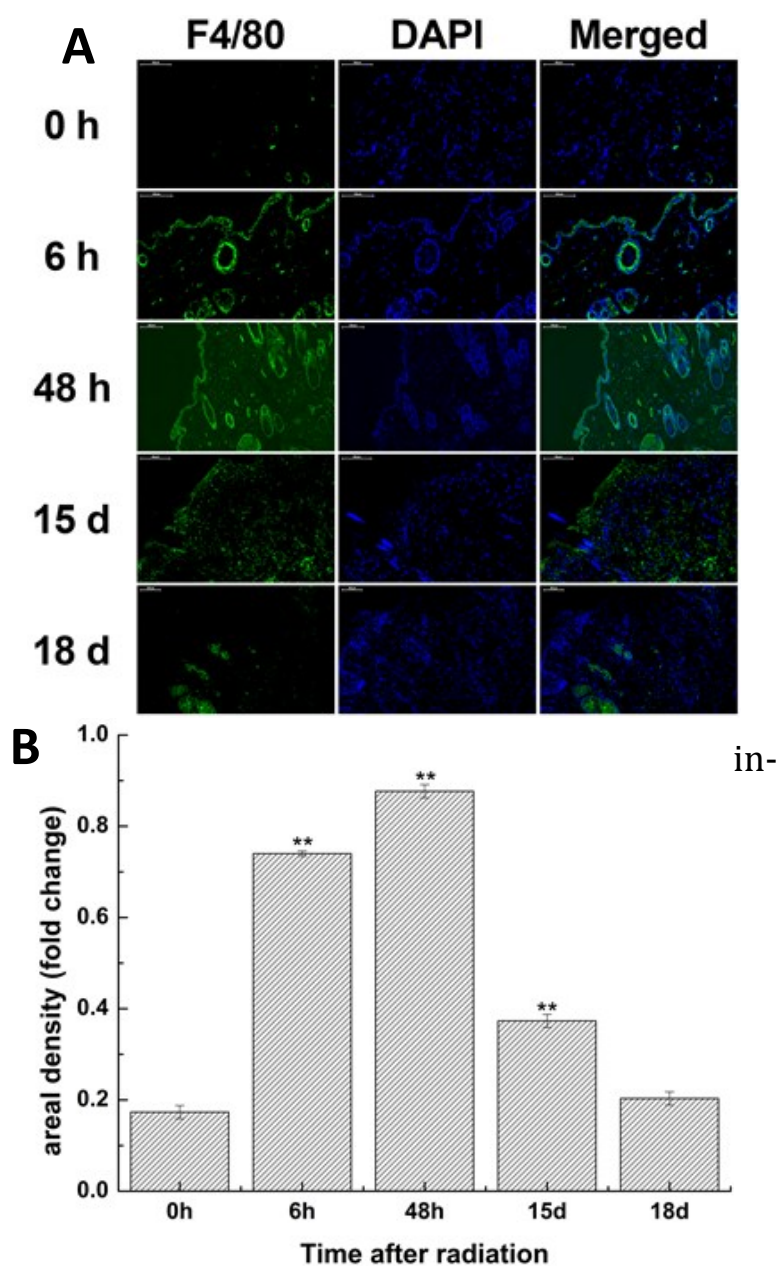

Figure 2. Immunofluorescence results of macrophage (F4/80) (A) and its expression (B) in irradiated skin between the X-ray group and the control group at different times after irradiation (magnification $\times 100$ ). *: $\mathrm{P}<0.05, * *$ : $\mathrm{P}<0.01$.

creased after irradiation.

\section{The localization of activation of inflammasomes in irradiated skin}

In the normal skin tissues, $\mathrm{NLRP}_{3}$ was mainly expressed in corneum tissue and some skin appendices, such as sebaceous glands in the dermis, and the expression level was low. However, no expression was observed in other parts of the dermis and epithelial tissues. At 6 
$\mathrm{h}-48 \mathrm{~h}$ after irradiation, the expression of the $\mathrm{NLRP}_{3}$ protein increased when compared with the control group. The positive cells were mainly present in the epidermis and dermis, sebaceous glands, sweat glands, and other skin appendage, while the expression in other parts of dermis was slightly decreased. $\mathrm{NLRP}_{3}$ protein expression peaked at $48 \mathrm{~h}$ after irradiation. At $15 \mathrm{~d}$ after irradiation, $\mathrm{NLRP}_{3}$ protein expression decreased slightly. In addition to the hair follicle and basal layer, there was higher positive cells expression in dermal tissue. At $18 \mathrm{~d}$ after irradiation, $\mathrm{NLRP}_{3}$ protein expression decreased significantly, and the expression site was mainly observed in the basal part of the epidermis, however, expression in the dermis was negative (figure 3A, B).

The expression pattern and localization of the ASC protein was nearly identical to that of $\mathrm{NLRP}_{3}$, except that the expression level in normal tissues was lower when compared to that of $\mathrm{NLRP}_{3}$, but higher than that of $\mathrm{NLRP}_{3}$ at other time points. At $18 \mathrm{~d}$ after irradiation, ASC protein expression decreased, but it was still higher when compared to the control group. Caspase-1 was mainly located in the deep dermis and some skin appendices in the normal skin tissues, but not in the superficial dermis and epidermal tissues. At $6 \mathrm{~h}-48 \mathrm{~h}$ after irradiation, the expression of caspase- 1 protein increased when compared with the control group. The positive cells were not only present in the deep dermal tissues and skin appendages, but also in the epidermal tissues, and expression peaked at $48 \mathrm{~h}$ after irradiation. At $15 \mathrm{~d}$ after irradiation, the number of positive caspase- 1 cells was still high, and the location of the positive cells was identical to that at $6 \mathrm{~h}-48 \mathrm{~h}$ after irradiation. At $18 \mathrm{~d}$ after irradiation, the expression of caspase- 1 protein decreased. In addition to the target protein-positive cells in the epidermis, caspase- 1 positive cells were observed in the deep dermis, however, protein expression was lower when compared to that in the control group (figure 3A, B).

After irradiation, expression of $\mathrm{NLRP}_{3}, \mathrm{ASC}$, and caspase- 1 protein in irradiated skin increased, and peaked at $48 \mathrm{~h}$ after irradiation,

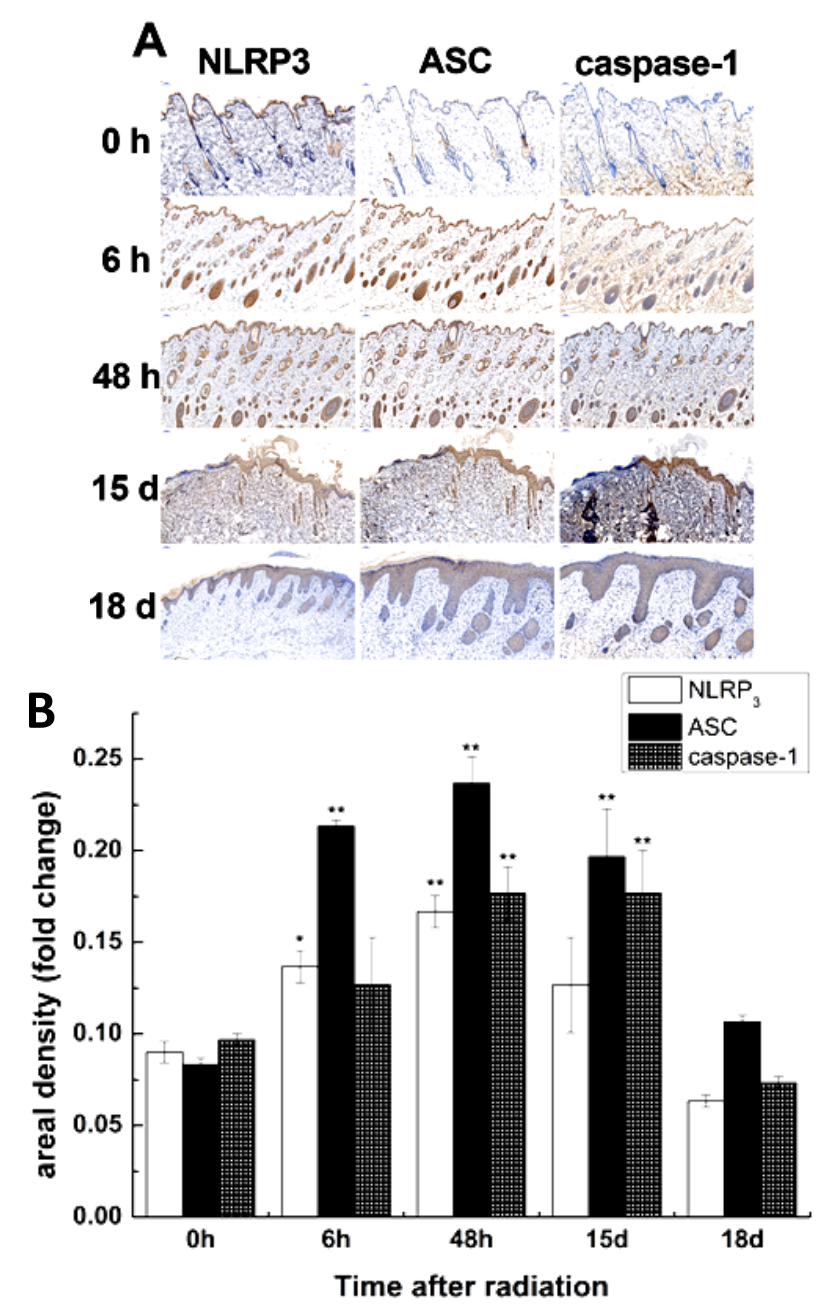

Figure 3. Immunohistochemical results of NLRP3, ASC, and caspase-1 (A) and their protein expression (B) in irradiated skin between the $X$-ray group and the control group at different times after irradiation (magnification $\times 20)$. *: $\mathrm{P}<0.05, * *: \mathrm{P}<0.01$.

which proved that the acute radioactive skin injury model was successfully established.

The DNA damage and inflammatory related cytokines expression in non-irradiated area-thymus

At $6 \mathrm{~h}-48 \mathrm{~h}$ after irradiation, $\gamma \mathrm{H}_{2} \mathrm{AX}$ mRNA, and protein expression in rat thymus continued to increase, and statistically significant differences were observed with the control group $(\mathrm{P}<0.01)$. At $15 \mathrm{~d}$ after irradiation, when the wound was most severe, $\gamma \mathrm{H}_{2} \mathrm{AX}$ mRNA and protein expression reached a peak $(\mathrm{P}<0.01)$. At $18 \mathrm{~d}$ after irradiation, $\gamma \mathrm{H}_{2} \mathrm{AX}$ mRNA expression decreased, but was higher when compared to 

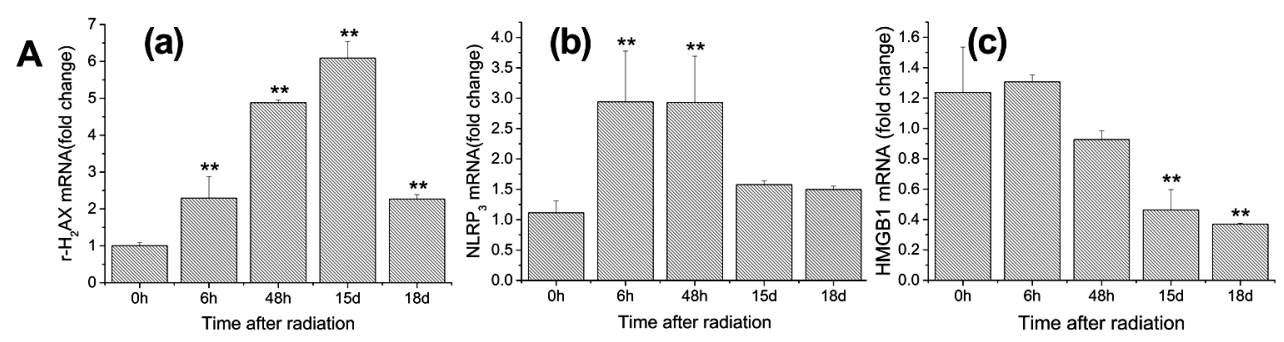

B
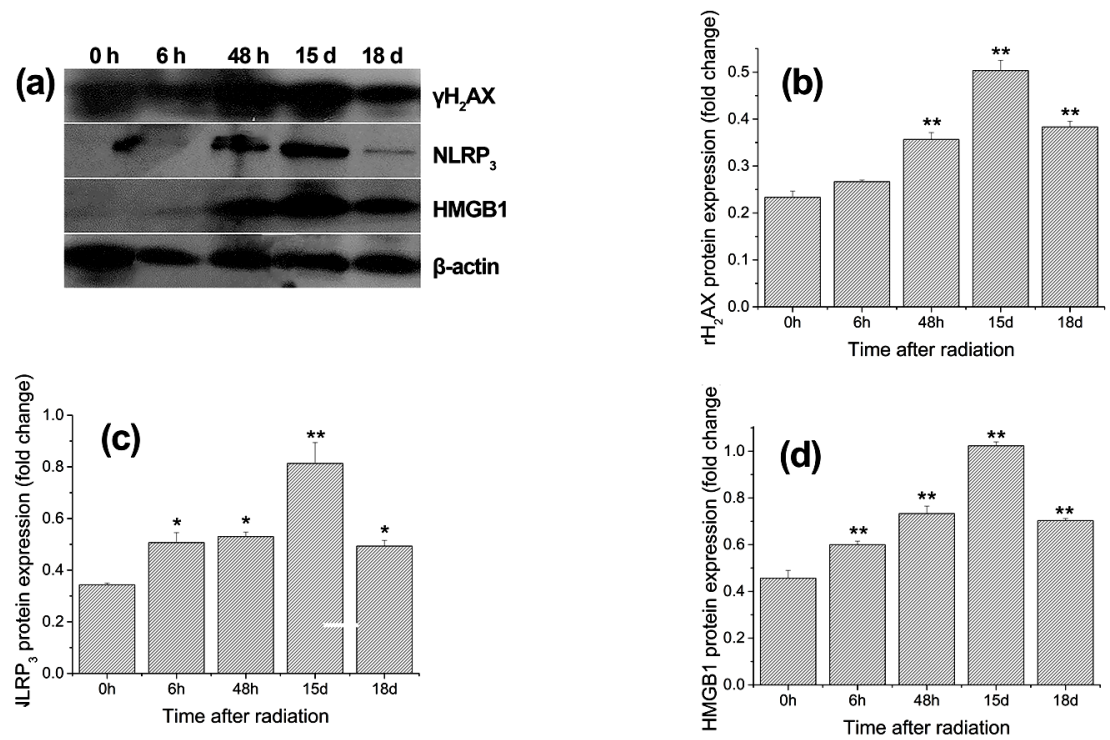

Figure 4. (A) qRT-PCR for $\mathrm{YH} 2 \mathrm{AX}$ (a), NLRP3 (b) and HMGB1 (c) was performed between the X-ray group and the control group at different times after irradiation. (B) (a) Representative Western blot results for $\mathrm{HH} 2 \mathrm{AX}, \mathrm{NLRP3}$, and HMGB1. (b-d) Statistical analysis of the Western blot results for $\mathrm{YH} 2 \mathrm{AX}, \mathrm{NLRP3}$, and HMGB1 between the X-ray group and the control group at different times after irradiation. Results were expressed as the mean \pm SEM ( $n=6$ per group). Significant differences were observed. ${ }^{*}: P<0.05, * *: P<0.01$. HMGB1= high mobility group box1.

the control group $(\mathrm{P}<0.01)$ (figure $4 \mathrm{~A}(\mathrm{a})$, figure 4B (b)).

At $6 \mathrm{~h}$ after irradiation, $\mathrm{NLRP}_{3}$ mRNA, and protein expression in rat thymus reached a peak $(\mathrm{P}<0.01)$. At $48 \mathrm{~h}$ after irradiation, $\mathrm{NLRP}_{3} \mathrm{mRNA}$ expression was nearly identical to that at $6 \mathrm{~h}$ after irradiation $(\mathrm{P}<0.01)$, while the protein expression was slightly higher when compared to that at $6 \mathrm{~h}$ after irradiation $(\mathrm{P}<0.01) . \mathrm{NLRP}_{3}$ protein expression reached its peak at $15 \mathrm{~d}$ after irradiation, however, the mRNA expression decreased when compared with that at $48 \mathrm{~h}$ after irradiation. At $18 \mathrm{~d}$ after irradiation, the mRNA expression was not significantly different from the control group ( $\mathrm{P}>0.05)$, however, the protein expression was slightly higher when compared to the control group $(\mathrm{P}<0.05)$ (figure $4 \mathrm{~A}(\mathrm{~b})$, figure $4 \mathrm{~B}(\mathrm{c}))$.

At $6 \mathrm{~h}$ after irradiation, HMGB1 mRNA expression increased slightly, however, no significant differences were observed when compared with the control group $(\mathrm{P}>0.05)$. Furthermore, protein expression was increased $(\mathrm{P}<0.01)$. At $48 \mathrm{~h}$ after irradiation, HMGB1 mRNA expression decreased $(\mathrm{P}>0.05)$, while the protein expression continued to increase $(\mathrm{P}<0.01)$. The HMGB1 mRNA expression continued to decrease $(\mathrm{P}<0.01)$, while the protein expression continued to increase $(\mathrm{P}<0.01)$ at $15 \mathrm{~d}$ after irradiation. At $18 \mathrm{~d}$ after irradiation, HMGB1 mRNA and protein expression decreased, however, there was still a significant difference when compared with the control group $(\mathrm{P}<0.01)$ (figure $4 \mathrm{~A}(\mathrm{c})$, figure $4 \mathrm{~B}$ (d)).

After radiation was applied to skin tissues, the DNA damage related-factor $\gamma \mathrm{H}_{2} \mathrm{AX}$ in the rat thymus was activated and stimulated inflammatory response. Moreover, the protein expression of the three factors followed the 
same trend, and the expression was the highest at $15 \mathrm{~d}$ after irradiation.

DNA damage and inflammatory-related cytokines expression in non-irradiated area-thymus after dosing

HMGB1 increased the mRNA and protein expression of $\gamma \mathrm{H}_{2} \mathrm{AX}$ in rat thymus when compared with the control group $(\mathrm{P}<0.01)$ and the $15 \mathrm{~d}$ group after irradiation $(\mathrm{P}<0.05)$. IN addition, GA significantly increased the mRNA and protein expression of $\gamma \mathrm{H}_{2} \mathrm{AX}$ when compared with the control group $(\mathrm{P}<0.01)$, however decreased mRNA $(\mathrm{P}>0.05)$ and protein expression $(\mathrm{P}<0.01)$ were observed when compared with the $15 \mathrm{~d}$ group after irradiation (figure 5A (a), figure 5B (b)).

HMGB1 significantly increased the mRNA and protein expression of $\mathrm{NLRP}_{3}$ in rat thymus when compared with the control group and the $15 \mathrm{~d}$ group after irradiation $(\mathrm{P}<0.05)$. Furthermore, GA increased the mRNA and protein expression when compared with the control group $(\mathrm{P}<0.01)$. GA solution increased the mRNA expression $(\mathrm{P}<0.01)$, but decreased the protein expression when compared with the $15 \mathrm{~d}$ group after irradiation $(\mathrm{P}<0.01)$ (figure $5 \mathrm{~A}(\mathrm{~b})$, figure $5 \mathrm{~B}(\mathrm{c}))$.

HMGB1 increased the HMGB1 mRNA and protein expression $(\mathrm{P}<0.01)$ compared with the control group and the $15 \mathrm{~d}$ group after irradiation $(\mathrm{P}<0.01)$. GA decreased the HMGB1 mRNA expression $(\mathrm{P}<0.01)$, but increased the protein expression $(\mathrm{P}<0.01)$ compared with the control group. GA solution decreased the HMGB1 mRNA and protein expression when compared with the $15 \mathrm{~d}$ group after irradiation $(\mathrm{P}<0.01)$ (figure 5A (c), figure 5B (d)).

At $15 \mathrm{~d}$ after skin irradiation, when the skin wound was characterized as the most severe, pro-inflammatory and anti-inflammatory drugs were given. One day later, rats treated with the pro-inflammatory drug HMGB1 showed increased expression of DNA damage-related factor $\gamma \mathrm{H}_{2} \mathrm{AX}$, inflammatory-factor $\mathrm{NLRP}_{3}$, and HMGB1. Rats treated with the anti-inflammatory (a)

Figure 5. (A) qRT-PCR for $\gamma \mathrm{H} 2 \mathrm{AX}(\mathrm{a}), \operatorname{NLRP3}(\mathrm{b})$, and HMGB1 (c) was performed between the $X$-ray group, the $X$-ray+drug group and the control group. (B) (a) Representative Western blot results for $\mathrm{YH} 2 \mathrm{AX}$, NLRP3, and HMGB1. (b-d) Statistical analysis of the Western blot results for $\mathrm{YH} 2 \mathrm{AX}, \mathrm{NLRP} 3$, and HMGB1 between the $X$-ray group, the $X$-ray+drug group, and the control group. Results are expressed as the mean \pm SEM ( $n=6$ per group). Significant differences were observed. *: P<0.05, **: $\mathrm{P}<0.01$. $\mathrm{N}=$ non-irradiated control. HMGB1= irradiation+high mobility group box1. GA= irradiation+glycyrrhizic acid. $\mathrm{C}=$ at $15 \mathrm{~d}$ after irradiation.

B (a)

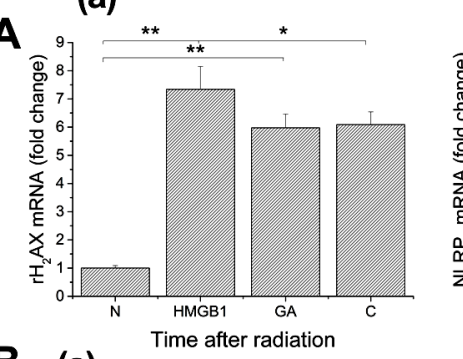

(b)

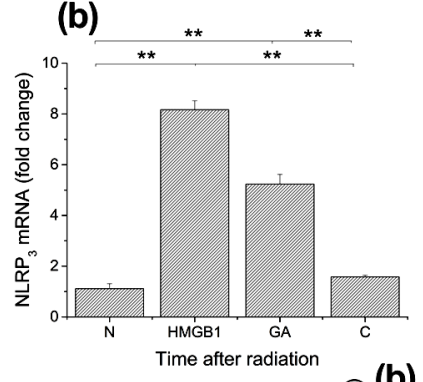

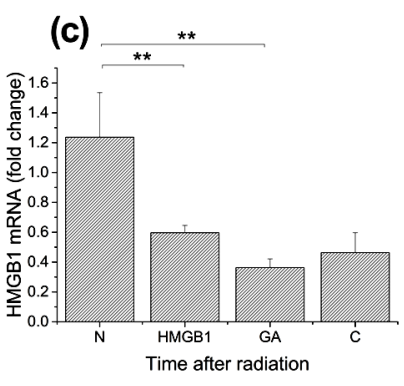
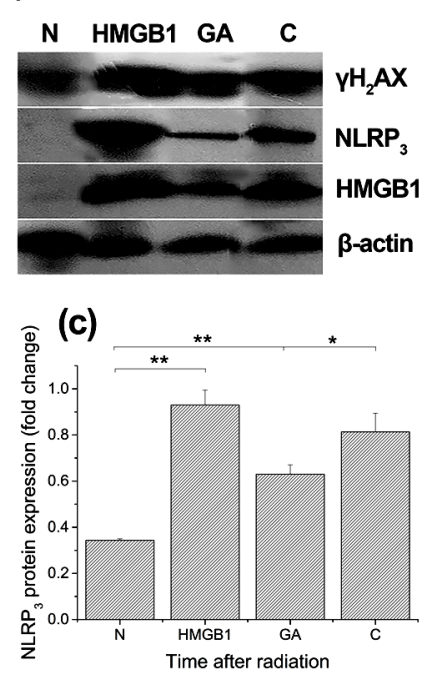
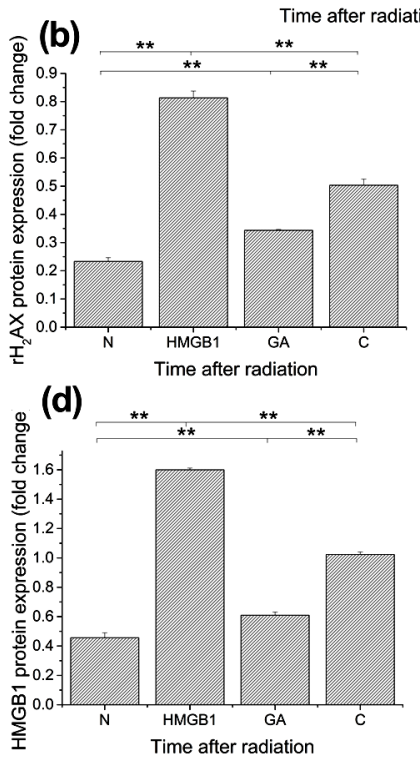
drug GA, showed decreased expression of these three factors.

\section{DISCUSSION}

Since the discovery of X-ray, ionizing radiation has been widely used in the diagnosis and treatment of clinical diseases, especially the rapid development of radiotherapy. Previously, DNA damage directly induced by ionizing radiation or not properly repaired by cells was generally regarded as the direct cause of biological effects, such as cell apoptosis, micronucleus formation, abnormal growth mutations, and carcinogenesis. In 1992, Nagasawa and Little (2) found that in a monolayer of cultured cells, the nuclei of less than $1 \%$ of cells were penetrated by single alpha rays, while over $30 \%$ of cells showed an increased frequency of sister chromatid exchange, which was the first introduction of the definition of radiation-induced bystander effects. Bystander effects have been demonstrated both in vitro and in vivo when using high linear energy transfer rays, such as $\alpha$-rays, heavy particles or low linear energy transfer (LET) rays, such as X-rays and $\gamma$-rays on different cells $(18,19)$, and their underlying mechanism of action has been preliminarily explored. However, since the discovery of the phenomenon, the exact mechanism has not yet been revealed.

The 38 Gy dose selected in this experiment were similar to the doses used in clinical patients. For example, the skin damage state of rats exposed to a 40 Gy radiation dose was similar to the skin condition of patients with a cumulative radiation dose of 16 Gy to $20 \mathrm{~Gy}$ (20). In this study, it was found that in the first two weeks after irradiation, the skin of the rats appeared slightly red and there was some bleeding, but no obvious inflammatory responses were observed under the microscope. However, after 14 days, especially at $15 \mathrm{~d}$ after irradiation, the skin tissue was ruptured with exudate, and large areas of epidermal cell necrosis could be observed microscopically, indicating an acute inflammatory response. The expression of macrophage (F4/80) increased at $6 \mathrm{~h}$ after irradiation, and at $15 \mathrm{~d}$ after irradiation, F4/80 was mainly distributed in the dermis, thereby suggesting that F4/80 was activated after irradiation, as were inflammatory factors $\mathrm{NLRP}_{3}$ and IL-1 $\beta$ in the blood which were previously studied by our team. Macrophages are key mediators of inflammation in the body. In the bystander condition, macrophages became activated and produced continuously increasing levels of oxidative stress after radiation exposure (21), which was followed by the secretion of cytokines. Cytokines, in turn, can reach distant tissues, oxidative stress can form reactive oxygen species (ROS), and causing DNA damage that leads to an inflammatory response by regulating inflammatory factors in the blood, which was consistent with the results of Jessica et al. (22).

The thymus is an important part of the body's immune system. It is the central lymphoid organ of $\mathrm{T}$ cell development, differentiation, and maturation. Lymphocytes from the thymus are very sensitive to radiation. It is well-known that after a high dose of radiation, DNA in the body's immune cells will be double-stranded, and severe DNA damage will lead to apoptosis and even death. Pabst etal. (23) found that many apoptotic lymphocytes appeared in the thymus of rats at $1 \mathrm{~d}$ after $\gamma$-ray irradiation. At $6 \mathrm{~h}$ after irradiation, the first injury recovered, and the second injury started again at $28 \mathrm{~d}$ after irradiation. Some believed that the thymus stromal cells promoted the repair of thymic tissue (24), while others believed that lymphocytes proliferated under the action of pro -inflammatory factors (25). The results of this study showed that the expression of $\gamma \mathrm{H}_{2} \mathrm{AX}$, $\mathrm{NLRP}_{3}$, and HMGB1 in the thymus of rats peaked at $48 \mathrm{~h}-15 \mathrm{~d}$ after irradiation, however, according to the immunohistochemical results, inflammatory factors in the skin of the irradiated area peaked at $48 \mathrm{~h}$ after irradiation. These findings indicated that the inflammatory response in the irradiated skin area first reached the peak, then the inflammation in other organs reached the peak. In addition, studies on the mechanisms underlying RIBE have identified possible signaling pathways. Further studies can be conducted on inflammatory sub-pathways, including caspase-1, IL-1 $\beta$, and transforming 
growth factor beta (TGF- $\beta 1$ ), $\alpha$-smooth muscle actin ( $\alpha$-sma), as well as other related pathways, such as MAPK, NF-kB /Cox2, to promote improvement of the radiation treatment effect, and reduce adverse reactions. Moreover, HMGB1 significantly increased the expression of $\gamma \mathrm{H}_{2} \mathrm{AX}, \mathrm{NLRP}_{3}$ and HMGB1 in rat thymus, and GA decreased expression of $\gamma \mathrm{H}_{2} \mathrm{AX}, \mathrm{NLRP}_{3}$ and HMGB1. Our data showed that the radiation effects on the skin and circulation to the thymus tissues, resulted in thymus injury, which was necessary to explore opportunities for radiation-induced systemic effects to be mediated by antioxidants, anti-inflammatory drugs, or agents that affect pathways that cause bystander effect instability.

Previous studies were mostly focused on the cell and tissue level and the time nodes were selected narrowly. Animal models were limited and the time span was not long. In this study, a single high-dose of X-ray was used to irradiate the skin of the buttocks of rats to establish an acute radiation skin injury model. We found that activation of macrophages in the irradiated skin caused cytokine production, then cytokines activated distant organ (the thymus) via the circulation, which caused DNA damage and inflammation-related cytokines in the non-irradiated thymus. Moreover, rats were injected with proinflammatory and anti-inflammatory drugs at $15 \mathrm{~d}$ after irradiation, which showed and increase and decrease in expression, respectively, of $\gamma \mathrm{H}_{2} \mathrm{AX}$, $\mathrm{NLRP}_{3}$ and HMGB1. These findings have a significant and constructive effect on the clinical radiotherapy of tumors, and in further studies, the underlying mechanism of RIBEs will be better understood, and applied to the practical work of radiotherapy and protection. Thus, an appropriate dose of anti-inflammatory drugs can reduce the RIBEs that reduces damage to distant parts of the patient.

\section{ACKNOWLEDGEMENTS}

This work was partially supported by the National Natural Science Foundation of China [31870934], University Science and Technology 418
Innovation Project of Shanxi Province [2019L0256], a Project Funded by Shanxi Key Laboratory of Material Strength \& Structural Impact [sxmssi-201802], and a Project Funded by Beijing Letang Life Technology Co., Ltd. [RH1900001846].

Conflicts of interest: Declared none.

\section{REFERENCES}

1. Desouky O, Ding N, Zhou G (2015) Targeted and nontargeted effects of ionizing radiation. J Radiat Res App/ Sc, 8: 247-254.

2. Nagasawa H and Little JB (1992) Induction of sister chromatid exchanges by extremely low doses of alphaparticles. Cancer Res, 52: 6394-6396.

3. Wang HZ, Yu KN, Hou J, Liu Q, Han W (2015) Radiationinduced bystander effect: early process and rapid assessment. Cancer Lett, 356: 137-144.

4. Rasola A and Bernardi P (2007) The mitochondrial permeability transition pore and its involvement in cell death and in disease pathogenesis. Apoptosis, 12: 815-833.

5. Liu SZ, Jin SZ, Liu XD, Sun YM (2001) Role of CD28/B7 costimulation and IL-12/IL-10 interaction in the radiationinduced immune changes. BMC Immunol, 2: 1-8.

6. Yuichiro Y, Ayako N, Sakamoto (2018) The moss physcomitrella patens is hyperresistant to DNA double-Strand Breaks induced by $\gamma$-irradiation. Genes, 9: 76.

7. Wilson TE and Sunder S (2020) Double-strand breaks in motion: implications for chromosomal rearrangement. Curr Genet, 66:1-6.

8. Rothkamm K, Barnard S, Moquet J, Ellender M, Rana Z, Burdak-Rothkamm S (2015) DNA damage foci: Meaning and significance. Environ Mol Mutagen, 56: 491-504.

9. Lumniczky K, Candéias SM, Gaipl US, Frey B (2018) Editorial: Radiation and the Immune System: Current Knowledge and Future Perspectives. Front Immunol, 8: 1-3.

10. Xin $\mathrm{H}$ and Ji YX (2019) Effect of co-administration of Linggui zhugan decoction and Western medicine on inflammatory cytokines, and immune and cardiac functions of patients with chronic heart failure. Trop J Pharm Res, 18: 365-370.

11. Martinon F (2002) The inflammasome: a molecular platform triggering activation of inflammatory caspases and processing of pro-IL-beta. Mol Cell, 15: 10-18.

12. Sutterwala FS, Haasken S, Cassel SL (2014) Mechanism of $\mathrm{NLRP}_{3}$ inflammasome activation. Ann NY Acad Sci, 1319: 82-95.

13. Yang $\mathrm{H}$, Liu $\mathrm{H}$, Zeng $\mathrm{Q}$, Imperato $\mathrm{GH}$, Addorisio ME, Li JH, He MJ, Cheng KF, Al-Abed Y, Harris HE, Chavan SS (2019) Inhibition of HMGB1/RAGE-mediated endocytosis by HMGB1 antagonist box A, anti-HMGB1 antibodies, and

Int. J. Radiat. Res., Vol. 19 No. 2, April 2021 
cholinergic agonists suppresses inflammation. Mol Med Rep, 25: 1-13.

14. Khan PA, Thube R, Rab RA (2014) Formulation development and evaluation of silymarin gel for psoriasis treatment. J Innov Pharm Biol Sci, 1: 21-26.

15. Burdak-Rothkamm S and Rothkamm K (2018) Radiationinduced bystander and systemic effects serve as a unifying model system for genotoxic stress responses. Mytat ResRev Mutat, 778: 13-22.

16. Kim EJ, Park SY, Baek SE, Jang MA, Lee WS, Bae SS, Kim K, Kim CD (2018) HMGB1 increases IL-1 $\beta$ production in vascular smooth muscle cells via $\mathrm{NLRP}_{3}$ inflammasome. Front Physiol, 9: 313.

17. Hannah A, Agnieszka S, Helena EH, Peter L (2018) Ligation of free HMGB1 to TLR2 in the absence of ligand is negatively regulated by the $\mathrm{C}$-terminal tail domain. $\mathrm{Mol} \mathrm{Med}$, 24: 19.

18. Kanagaraj VR, Badri NP, Thayalan K, Venkatachalam P (2019) Primary and secondary bystander effect and genomic instability in cells exposed to high and low linear energy transfer radiations. Int J Radiat Biol, 95: 1648-1658.

19. Dong C, He MY, Tu WZ, Konishi T, Liu W, Xie YX, Dang BR, Li WJ, Uchihori Y, Hei TK, Shao, C. (2015) The differential role of human macrophage in triggering secondary by- stander effects after either gamma-ray or carbon beam irradiation. Cancer Lett, 363: 92-100.

20. Harper JL, Franklin LE, Jenrette JM, Aguero EG (2004) Skin toxicity during breast irradiation: pathophysiology and management. South Med J, 97: 989-993.

21. Havaki S, Kotsinas A, Chronopoulos E, Kletsas D, Georgakilas A, Gorgoulis VG (2015) The role of oxidative DNA damage in radiation induced bystander effect. Cancer Lett, 356: 43-51.

22. Jessica V, Pavel NL, Jason SP, Helen F, Nicole MH, Alesia I, Andrew WS, Christopher JH, Andreas N, Vasilis K, Gerasimos $C P$, Gianna $P$, Konstantinos $S$, Georgia $T$, loannis $S P$, Vassilis GG, Alexandros GG, Carl NS, Olga AM (2017) Localized Synchrotron Irradiation of Mouse Skin Induces Persistent Systemic Genotoxic and Immune Responses. Cancer Res, 77: 6389-6399.

23. Pabst $R$ (2019) The thymus is relevant in the migration of mature lymphocytes. Cell Tissue Res, 376: 19-24.

24. Zhou WF, Xu ZQ, Zhao KQ, Xu JJ, Dong Y, Tong SL (2019) Efficacy of human bone marrow mesenchymal stem cell transplantation in repair of radiation-induced damage to the immune system. Int J Clin Exp Med, 12: 2651-2658.

25. Pourgholaminejad A., Aghdami N, Baharvand H, Moazzeni SM (2016) The effect of pro-inflammatory cytokines on 
